Review Article

\title{
Evaluating equilibrium in anterior cruciate ligament reconstruction
}

\author{
Forough Ferdowsi, PT, $\mathrm{PhD}^{1)}$, Zahra Sadat Rezaeian, PT, $\mathrm{PhD}^{2)^{*}}$ \\ 1) Physical Therapy Department, School of Rehabilitation, Tehran University of Medical Sciences and \\ Health Services, Iran \\ 2) Musculoskeletal Research Center, Rehabilitation Sciences Research Institute, Department of \\ Physical Therapy, Faculty of Rehabilitation Sciences, Isfahan University of Medical Sciences: \\ Isfahan, PO Box: 73461-81746, Iran
}

\begin{abstract}
Purpose] The purpose of this review was to summarize available methods and equipment which are administered to evaluate the balance in anterior cruciate ligament reconstruction. [Subjects and Methods] A literature search was performed and reviewed using the narrative approach. This study reviewed English articles concerning balance assessment methods in anterior cruciate ligament reconstruction subjects from 1985 to 2015 using the following key words: Anterior Cruciate Ligament Injury, Anterior Cruciate Ligament Reconstruction, Postural Control, Equilibrium, Balance and Stability. [Results] This review included 44 studies out of 117 initially retrieved articles. These articles were discussed in balance measurement procedure without comparing the effect of various surgical, medical or rehabilitation approaches. [Conclusion] Biodex and EquiTest, functional dynamic tests are of choice in the evaluation and tracking of anterior cruciate ligament reconstruction subjects. Force plate parameters provide information regarding strategies of static postural control and is not sensitive enough to challenge postural control system in physical activities. Key words: Anterior cruciate ligament injury, Anterior cruciate ligament reconstruction, Postural control
\end{abstract}

(This article was submitted Aug. 12, 2017, and was accepted Feb. 27, 2018)

\section{INTRODUCTION}

The anterior cruciate ligament (ACL) of the knee is the principal factor controlling knee forward movement ${ }^{1)}$ whereas it is the most commonly injured ligament of the $\mathrm{knee}^{2)}$. Seventy percent of these injuries are non-contact ones which occurred as a result of rotation, sudden change in direction, jumping and falling ${ }^{3)}$.

ACL injury is a fundamental problem among active and mature youths which in average causes 11,300 outpatient and 7,500 ACL reconstruction surgeries. ACL is rich in mechanical receptors and is known as somatosensory organ in postural control $^{4}$. Thus, ACL injury causes joint instability and giving away episodes during the activities, and may be followed by limitation in physical and sport activity and low quality of life ${ }^{5,6)}$. Postural control deficiency is reported following ACLR ${ }^{7}$. Biomechanical changes ${ }^{8)}$, changes in quadriceps muscles $\left.{ }^{7}, 9\right)$ and brain activities and proprioception disorders ${ }^{9)}$ have been reported following ACLR. As the main criterion for the ACL reconstruction (ACLR) surgery, the individual's ability to return to the activities of daily living (ADL) and to the recreational and sports routines are the main concerns for those who suffer from ACL injury and is being considered ${ }^{10)}$.

Various studies have investigated the mechanism of equilibrium deficiency following ACLR ${ }^{7,8,11)}$; some showing an increase in postural sway and some showing no change ${ }^{9)}$ although no comprehensive study to date has investigated the available methods for evaluating equilibrium after ACLR. With regard to the present body of literature on the equilibrium after ACLR, the aim of the present study is to review all the methods applicable in studying equilibrium following ACLR.

*Corresponding author. Zahra Sadat Rezaeian (E-mail: zrezaeian@rehab.mui.ac.ir; zrezaeian@yahoo.com) (C2018 The Society of Physical Therapy Science. Published by IPEC Inc.

(c) $(-)$ This is an open-access article distributed under the terms of the Creative Commons Attribution Non-Commercial No Derivatives

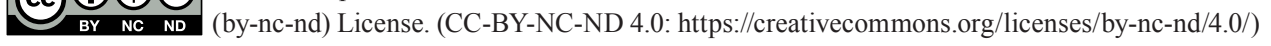




\section{SUBJECTS AND METHODS}

We searched MEDLINE and allied health literature for studies from 1985 through 2015 by using the following Medical Subject Heading (MeSH) terms and keywords: Anterior Cruciate Ligament Injury, Anterior Cruciate Ligament Reconstruction, Postural Control, Equilibrium, Balance and Stability. All original and review articles were included when they were written in English and when they were addressed using balance assessment methods in ACL injury and ACLR patient. The article may be included only if they were studying the variation in ACLR subjects without including medical or physical management interventions. Thus, the studies about kinds of ACL surgery, those comparing various rehabilitation or medical management strategies, and the studies of the effect of physical training procedures were not included in this review. Instead, case control studies, cohort studies and evaluation records were included. References of included studies were checked for additional studies meeting the inclusion criteria. A total of 44 studies were found with relevant data on our aim. These studies were categorized according to our kinds of balance assessment methods and reviewed in a narrative manner.

\section{RESULTS}

The primary search with keywords provided 106 articles. Moreover, 11 articles were found from communication with content experts in the field of ACL screening and rehabilitation in the 2010-2015 conference proceedings of the American College of Sports Medicine and European Society of Sports Traumatology, Knee Surgery, and Arthroscopy. From these 117 articles, 70 articles were excluded for not meeting the inclusion criteria, and 27 articles were excluded after comprehensive review of their method section. Finally, 20 full text articles were included in the present study.

\section{DISCUSSION}

Balance and postural control: Balance and postural control is a basic component of all motor skills. It is a complex function and a cognitive process ${ }^{12}$ ) that requires integrated coordination of sensory inputs from vestibular, visual and somatosensory systems. Insufficiency of any of these system results in balance impairment and postural control problems ${ }^{5)}$.

Mechanisms of BPC: Neural components of BPC are subdivided into: sensory, motor and cognitive sections. Cognitive part moderates the function of sensory and motor sections function response to imposed tasks or environmental changes and prepares them to deal with the changes ${ }^{2)}$.

Methods to evaluate the balance: Different studies have used various methods to assess BPC. Objective and variables of a study are the key criteria to select BPC measurement methods and interpret its findings.

Force plate (FP): Static postural control is the ability to maintain position in single and double limb stance and is presented by measuring displacement, velocity, acceleration and frequency of displacement of center of pressure (COP). In quiet standing posture, the body is fairly immobile and COP has a form of rocking motions named sway. Force plate records COP location in space at any time and help the assessor to evaluate subject's ability in maintaining COM inside BOS ${ }^{13)}$. The force plate data is used to calculate variability and regulation of position, in order to determine instability in maintaining static posture ${ }^{14)}$. However, force plate is not sensitive enough to challenge postural control system in physical activities ${ }^{15}$.

Biodex stability system (BSS): BSS consists of a multi-axial platform. This system identifies subject's ability to maintain dynamic stability on an unstable inclined platform. With selecting instability level, the subjects try to maintain the platform. Instability level is adjustable between the most stability level, level eight and the least instability level, level two. BBS produces three indices electronically which are named based on the degree of AP and mediolateral (ML) slop. The indices are anteroposterior stability index (APSI), mediolateral stability index (MLSI) and overall stability index (OSI). These mechanical tests could not challenge falls due to external perturbations during physical activity ${ }^{16)}$.

Equitest system: Equitest system is used to produce perturbations beyond BOS and measure balance reactions. It contains a pair of force plates (dual force plate) and produces perturbation of linear displacement (translatory) type. The system sets the displacement of support surface according to subject's height in a way that all the subjects get disturbed to the same angle (in degree) and with the angular moment in a defined period of time. The device screens data from sway (in degrees), shear force and COP and COM displacements in $\mathrm{X}$ and $\mathrm{Y}$ axes ${ }^{17)}$.

The role of ACL in balance and postural stability: Recent studies have provided strong evidences regarding sensory role of $\mathrm{ACL}^{8}$. It has even been proposed that proprioceptive neuro-physiological function of ACL is as important as its biomechanical function in the maintenance of joint stability ${ }^{10}$. If afferent information changes following ACL injuries, alteration in processing of cortical information is also expected. These changes have been confirmed by the study of evoked potentials and electroencephalography $(\mathrm{EEG})^{5)}$

ACL injury and its consequences in balance control process: Postural control is not normal following ACLD and ACLR ${ }^{7)}$. Numerous studies reported proprioception impairment after ACL injury ${ }^{18,19)}$. Impairment of afferent or efferent systems may damage balance control system; having this fact in mind that cognitive system is an-important component of the balance control system. Previous studies approved increased sway amplitude and frequency in ACLD ${ }^{1,7)}$ and ACLR ${ }^{7,8)}$ subjects compared to healthy individuals. These differences may be related to impairment in central mechanisms of postural control ${ }^{4,5,7)}$. 
Consequently, despite the fact that ACL injury is basically a peripheral musculoskeletal problem; it is currently mulled over a neurological dysfunction rather than a simple neuromuscular deficit ${ }^{20)}$.

In some studies, single limb stance (SLS) tests are used to measure subject's postural control ${ }^{21)}$. In ACL individuals, the sway is the same as standing on either sound or involve limb and is higher than that of healthy subjects. That means, ACLD affects both limbs irrespective of the injury side. This finding supports the central impairment theory ${ }^{7}$. That means peripheral injuries may involve central data processing system even without structural damage to processing pathways ${ }^{22}$.

Balance assessment methods in ACLR: A) Force plate (FP): Single limb balance seems not to be significantly different between sound and affected limb of 10-18 months post-ACL ${ }^{23)}$. However, it has been demonstrated that ACLR subjects have better balance standing on affected limb when compared to chronic ACLD subjects although the balance in both groups was significantly defective when compared to healthy individuals ${ }^{24,25)}$.

ACLR balance on sound and affected limb is controversial. In 1999, Haffman et al. ${ }^{9}$ evaluated static and dynamic balance in single limb support on FP in twenty ACLR individuals. They used electrical stimulation on tibia nerve to perturb subjects and measure their dynamic balance performance. The difference between ACLR and the control healthy group was statistically significant only in dynamic context. The required time to retrieve equilibrium following perturbation was significantly longer in ACLR group for both sound and reconstructed limbs. This finding implies that the affected side is neurologically in a mutual connection with sound side. This phenomenon accentuates the central mechanism of postural control and CNS plasticity to meet the functional needs of the subject ${ }^{9}$.

This way, the observed differences between loading rate of sound and affected limbs and between ACLR and normal subjects, proposed by Deker et al. ${ }^{21)}$ in 2002, is rational.

In 2012, Mohammadi et al. ${ }^{13}$ ) indicated that the sway was greater in single limb standing on reconstructed limb than that of sound limb and both were more than healthy controls.

Dingenen et al., evaluated postural stability during the transition from double-leg stance (DLS) to single-leg stance (SLS) in 20 ACLR and 20 healthy subjects. ACLR group showed postural stability deficits, indicating that these persons may have a decreased ability to stabilize their body after the internal postural perturbation created by the transition from DLS to SLS ${ }^{26)}$.

These researches all confirm that balance and balance control is impaired in ACLR subjects. In brief, balance and postural control impairment is one of the common consequences of ACLD and in some cases, it may not be completely resolved even months after reconstructive surgery.

B) Biodex indexes: Biodex indexes represent the accuracy of positional sense receptors in detecting BOS obliquity. They may also reflect the neuromuscular coordination to reach a dynamic stability despite standing on an unbalanced surface ${ }^{27)}$. Measuring APSI, MLSI and OSI confirmed that. ACLR may help in maintaining proprioception and functional stability of the knee joint. Although postural balance seems to be significantly lower in sedentary people and either limbs of ACLR clients and postural balance on affected limb and displacement of center of gravity (COG) may be less than that of sound limb in ACLR subjects ${ }^{28)}$.

C) Equitest system: Lysholm et al. studied 22 ACLD individuals using sensory organization and movement control test protocol in sagittal plane in Equitest. ACLDs show greater sway when standing on affected side on a stable surface with open eyes. Their sway was also higher than that of the control group in double standing with open eyes. Testing with closed eyes showed that ACLDs and normal subjects were not statistically different in double standing. Reaction time in double standing was longer in ACLDs with anteroposterior perturbations (motion control test) in comparison to normal group. In the same way, reaction time and sway rate in ACLD group were more than in healthy subjects in response to anteroposterior perturbation when standing on affected side. Balance impairment was documented in ACLD individuals when they were studied standing on either affected or sound side. The unexpected finding in the sound side has been explained by signals form static gamma motor neurons in a reflex activity in sound side ${ }^{1)}$.

Another study in 2001 showed that ACLRs have more sway in sensory organization test with closed eyes when they were tested in double stance on an unstable BOS, while they were not different from the control group in double standing on stable BOS. ACLRs single limb standing on stable BOS showed no difference between limbs. In movement control test, reaction time for anteroposterior perturbation was longer in ACLR individuals without any significant difference between the two limbs. Instead, control group had longer latency than ACLR group. Reaction time for lateral perturbation was not different between groups. This parameter was predictably longer than medial reaction time for both groups but no significant differences were recorded between the two groups and between the two limbs in each group. Medial fluctuating rates were more than lateral fluctuation without significant differences between inter and intra-group (between limbs) ${ }^{8}$.

Postural control is the basic needs of ADL. Balance dysfunction due to musculoskeletal injuries changes performance. ACLD and ACLR are very common in youth and impose considerable financial and social burden. Balance dysfunction assessment is critical in these people for planning a comprehensive rehabilitation protocol in early stage. ACL injury seems not to impose impaired postural control in static position because this position does not challenge the balance. Conversely, in real situation, several factors and perturbation challenge the balance system, therefore; dynamic assessments may better identify balance impairments. Accordingly, Biodex and Equitest, functional dynamic tests are of choice in the evaluation and tracking of these people.

A limitation of this review is its narrative approach. The included studies were not analyzed systematically and metaanalysis of the included articles was performed; therefore definitive conclusions cannot be drawn. A systematic review on present body of literature will help to provide a more clear benefit of available strategies. 


\section{Conflict of interest}

None.

\section{REFERENCES}

1) Lysholm M, Ledin T, Odkvist LM, et al.: Postural control—a comparison between patients with chronic anterior cruciate ligament insufficiency and healthy individuals. Scand J Med Sci Sports, 1998, 8: 432-438. [Medline] [CrossRef]

2) Ageberg E: Consequences of a ligament injury on neuromuscular function and relevance to rehabilitation-using the anterior cruciate ligament-injured knee as model. J Electromyogr Kinesiol, 2002, 12: 205-212. [Medline] [CrossRef]

3) Noyes FR, Barber SD, Mangine RE: Abnormal lower limb symmetry determined by function hop tests after anterior cruciate ligament rupture. Am J Sports Med, 1991, 19: 513-518. [Medline] [CrossRef]

4) Bonfim TR, Grossi DB, Paccola CA, et al.: Additional sensory information reduces body sway of individuals with anterior cruciate ligament injury. Neurosci Lett, 2008, 441: 257-260. [Medline] [CrossRef]

5) Courtney CA, Rine RM: Central somatosensory changes associated with improved dynamic balance in subjects with anterior cruciate ligament deficiency. Gait Posture, 2006, 24: 190-195. [Medline] [CrossRef]

6) Lee HM, Cheng CK, Liau JJ: Correlation between proprioception, muscle strength, knee laxity, and dynamic standing balance in patients with chronic anterior cruciate ligament deficiency. Knee, 2009, 16: 387-391. [Medline] [CrossRef]

7) della Volpe R, Popa T, Ginanneschi F, et al.: Changes in coordination of postural control during dynamic stance in chronic low back pain patients. Gait Posture, 2006, 24: 349-355. [Medline] [CrossRef]

8) Henriksson M, Ledin T, Good L: Postural control after anterior cruciate ligament reconstruction and functional rehabilitation. Am J Sports Med, 2001, 29: 359-366. [Medline] [CrossRef]

9) Hoffman M, Schrader J, Koceja D: An investigation of postural control in postoperative anterior cruciate ligament reconstruction patients. J Athl Train, 1999, 34: 130-136. [Medline]

10) Ferber R, Osternig LR, Woollacott MH, et al.: Gait perturbation response in chronic anterior cruciate ligament deficiency and repair. Clin Biomech (Bristol, Avon), 2003, 18: 132-141. [Medline] [CrossRef]

11) Brunetti O, Filippi GM, Lorenzini M, et al.: Improvement of posture stability by vibratory stimulation following anterior cruciate ligament reconstruction Knee Surg Sports Traumatol Arthrosc, 2006, 14: 1180-1187. [Medline] [CrossRef]

12) Akhbari B, Salavati M, Ahadi J, et al.: Reliability of dynamic balance simultaneously with cognitive performance in patients with ACL deficiency and after ACL reconstructions and in healthy controls. Knee Surg Sports Traumatol Arthrosc, 2015, 23: 3178-3185. [Medline] [CrossRef]

13) Mohammadi F, Salavati M, Akhbari B, et al.: Static and dynamic postural control in competitive athletes after anterior cruciate ligament reconstruction and controls. Knee Surg Sports Traumatol Arthrosc, 2012, 20: 1603-1610. [Medline] [CrossRef]

14) Harrison EL, Duenkel N, Dunlop R, et al.: Evaluation of single-leg standing following anterior cruciate ligament surgery and rehabilitation. Phys Ther, 1994, 74: 245-252. [Medline] [CrossRef]

15) Salavati M, Hadian MR, Mazaheri M, et al.: Test-retest reliability [corrected] of center of pressure measures of postural stability during quiet standing in a group with musculoskeletal disorders consisting of low back pain, anterior cruciate ligament injury and functional ankle instability. Gait Posture, 2009, 29: 460-464. [Medline] [CrossRef]

16) Mazaheri M, Salavati M, Negahban H, et al.: Postural sway in low back pain: Effects of dual tasks. Gait Posture, 2010, 31: 116-121. [Medline] [CrossRef]

17) Negahban H, Hadian MR, Salavati M, et al.: The effects of dual-tasking on postural control in people with unilateral anterior cruciate ligament injury. Gait Posture, 2009, 30: 477-481. [Medline] [CrossRef]

18) van Emmerik RE, van Wegen EE: On the functional aspects of variability in postural control. Exerc Sport Sci Rev, 2002, 30: 177-183. [Medline] [CrossRef]

19) Hertel JM, Denegar C: Intratester and intertester reliability during Star Excursion Balance Tests. J Sport Rehabil, 2000, 9: 104-116. [CrossRef]

20) Herrington L, Hatcher J, Hatcher A, et al.: A comparison of Star Excursion Balance Test reach distances between ACL deficient patients and asymptomatic controls. Knee, 2009, 16: 149-152. [Medline] [CrossRef]

21) Decker MJ, Torry MR, Noonan TJ, et al.: Landing adaptations after ACL reconstruction. Med Sci Sports Exerc, 2002, 34: 1408-1413. [Medline] [CrossRef]

22) Corrigan JP, Cashman WF, Brady MP: Proprioception in the cruciate deficient knee. J Bone Joint Surg Br, 1992, 74: 247-250. [Medline] [CrossRef]

23) Fischer-Rasmussen T, Jensen PE: Proprioceptive sensitivity and performance in anterior cruciate ligament-deficient knee joints. Scand J Med Sci Sports, 2000, 10: 85-89. [Medline] [CrossRef]

24) Swanik CB, Covassin T, Stearne DJ, et al.: The relationship between neurocognitive function and noncontact anterior cruciate ligament injuries. Am J Sports Med, 2007, 35: 943-948. [Medline] [CrossRef]

25) Broglio SP, Tomporowski PD, Ferrara MS: Balance performance with a cognitive task: a dual-task testing paradigm. Med Sci Sports Exerc, 2005, 37: 689-695. [Medline] [CrossRef]

26) Dingenen B, Janssens L, Claes S, et al.: Postural stability deficits during the transition from double-leg stance to single-leg stance in anterior cruciate ligament reconstructed subjects. Hum Mov Sci, 2015, 41: 46-58. [Medline] [CrossRef]

27) Mohammadirad S, Salavati M, Takamjani IE, et al.: Intra and intersession reliability of a postural control protocol in athletes with and without anterior cruciate ligament reconstruction: a dual-task paradigm. Int J Sports Phys Ther, 2012, 7: 627-636. [Medline]

28) Kim DK, Park WH: proprioceptive and strength comparison of remnant preserved versus conventional anterior cruciate ligament reconstruction. Korean J Sports Med, 2011, 29: 99-104. [CrossRef] 